\title{
Modelling Health Process and System Requirements Engineering for Better e-Health Services in Saudi Arabia
}

\author{
Fuhid Alanazi ${ }^{1}$, Valerie Gay ${ }^{2}$ \\ Faculty of Engineering and Information Technology \\ University of Technology Sydney, Australia
}

\author{
Mohammad N. Alanazi ${ }^{3}$ \\ College of Computer and Information Sciences \\ Al Imam Mohammad Ibn Saud Islamic University (IMSIU) \\ Riyadh, Saudi Arabia
}

\author{
Ryan Alturki ${ }^{4}$ \\ Department of Information Sciences \\ College of Computer and Information Systems \\ Umm Al-Qura University, Makkah, Saudi Arabia
}

\begin{abstract}
This systematic review aimed to examine the published works on e-health modelling system requirements and suggest one applicable to Saudi Arabia. PRISMA method was adopted to search, screen and select the papers to be included in this review. Google Scholar was used as the search engine to collect relevant works. From an initial 74 works, 20 were selected after all screening procedures as per PRISMA flow diagram. The 20 selected works were discussed under various sections. The review revealed that goal setting is the first step. Using the goals, a model can be created based on which system requirements can be elicited. Different research used different approaches within this broad framework and applied the procedures to varying healthcare contexts. Based on the findings, an attempt has been made to set the goals and elicit the system requirements for a diabetes self-management model for the entire country in Saudi Arabian context. This is a preliminary model which needs to be tested, improved and then implemented.
\end{abstract}

Keywords-e-health; e-health systems; e-healthy modelling and e-health modelling system requirements

\section{INTRODUCTION}

Healthcare is a necessity globally. A common adage says a healthy nation is a wealthy nation. This realization has called for massive investments in healthcare in many nations of the world (Alanezi, 2020). However, healthcare remains a luxury for many populations across the globe, and correcting that anomaly requires strategic interventions, including the adoption of technologies. Information and Communication Technologies (ICTs) have opened up possibilities for virtually all aspects of human endeavour. Everything is going digital. A common example that comes to mind is e-Commerce. For many years, research and health practitioners have toyed with the idea of developing e-health models for health systems across the world. An effective e-health system will ensure the effective coverage of multiple health interventions such as telemedicine, telehealth, mobile health, health records, big data and even artificial intelligence.

In the words of Adhikari (2019), e-Health will also encourage equity, ethical standards among practitioners and promote healthcare education among patients and consumers. While the immense potential benefits for man is well covered, developing a holistic model and modelling system requirements has proven difficult. As Salah, Omran and Lari (2018) suggests, the vision has met with challenges relating to the complexities of the healthcare sector, compatibility issues and the issues of security and unification of eHealth frameworks [20].

Therefore, attempts to incorporate a holistic model requirement in the universal health system has not yielded much success. Efforts aimed at arriving at operationalizing eHealth and enabling its implementation in healthcare practice, education, research and policy have often lacked sufficient conceptual clarity. This has led to the widespread call for a conceptual and practical model of e-Health which adequately captures potential overlaps and complexities (JMIR). In Saudi Arabia, the need to adopt sustainable health issuance strategies and break the barriers limiting the adoption of e-Health has been widely written about. Several e-Health modelling requirements have also been provided by healthcare management experts and scholars around the world, but adopting such within the context of Arabian healthcare would call for comprehensive studies.

This paper, therefore, undertakes a systematic review of eHealth modelling system requirements to explore the possibility of suggesting them for Saudi Arabian healthcare.

The methodology followed for this review is given next.

\section{Methodology OF SELECTING LiterAture FOR THIS REVIEW}

The Preferred Reporting Items for Systematic Reviews and Meta-Analyses (PRISMA) method was followed for search, screen and selection of literature for this review. Search terms, "e-Health", "e-Health systems", "e-Health modelling" and "eHealth modelling system requirements" were used to search for research work in Google Scholar using anytime and 2015 and beyond as the time frames. Totally, 74 works were obtained. Out of these, 12 duplicates and works on the same 
aspects reported twice and in two sources were removed. Six (6) works, done by the same set of authors on the same aspect over a few years were removed after selecting the most important latest works covering earlier results. When closely similar repetitive points coming from different sources were seen, only one or two representative ones were selected from them. This resulted in not including another 12 papers. Another 24 works were not relevant to the topic and hence excluded. Thus, 20 papers were finally selected for this review as shown in the PRISMA flow diagram in the APPENDIX.

\section{WHAT IS E-HEALTH AND ITS IMPORTANCE}

Information and Communication Technology (ICT) has several applications in various sectors like agriculture, industry and transportation. The health sector also can immensely benefit from application of ICT. When these technologies are applied to healthcare, such as improving quality of care, electronic patient records- EPR, selfmanagement of chronic diseases through access to information and remote care or accessibility of remote communities (telemedicine) and other fields of healthcare, it becomes ehealth. It is where electronic (digital) technologies meet health. The WHO briefly defines e-health as "the use of information and communication technologies (ICT) for health." (WHO, 2019).

On his part, Eysenbach (2001) defined e-health as "an emerging field in the intersection of medical informatics, public health and business, referring to health services and information delivered or enhanced through the Internet and related technologies. He adds that the term also takes on a broader meaning as it characterises not only a technical development, but also a state-of-mind, a way of thinking, an attitude and a commitment for networked, global thinking, to improve health care locally, regionally and worldwide by using information and communication technology". This definition seems to suggest that e-Health, when adopted, should not remain an innovation, but should become a way of live, deeply entrenched in the psyche and attitudes of people.

\section{MAIN COMPONENTS OF E-HEALTH}

Despite its simple definitions, e-Health is a broad and complex arrangement with several components under its wings. As Alanezi (2020) [11] puts it, these components include;

- Electronic Medical Coverage: These are also called electronic health records and refer to real-time health records of patients with clear access to decision-support tools which can aid clinicians in decision making. It also refers to patient and population health information, collected and stored systematically and electronically in a digital format (Gunter \& Terry,2005).

- Electronic Medical Records: This has quite a simple definition. This component refers to digital records kept by doctors for recording and tracking the health data and metrics of their clients and patients. The Morsani College of Medicine at the University of South Florida calls it the equivalent of charts and paper records that you'd otherwise find in a clinician's office or the hospital's registry.

- Telemedicine: The WHO report on the second e-Health global survey of 2010 describes telemedicine simply as "healing at a distance". At closer look, the global health body posits that it is a way to improve patient outcomes through increased access to medical information and care by use of ICT (WHO, 2010). Here, they say, "distance is a critical factor" and erasing the barriers of distance is the essence of telemedicine.

Others like Picture Archiving \& Communication Systems (PACSs) are also worthy of note. But it doesn't end there, Eysenbach (2001) suggests there are business concepts that also form components of e-Health and which must be considered. The author added that the business concepts of $\mathrm{B} 2 \mathrm{~B}, \mathrm{~B} 2 \mathrm{C}$ and $\mathrm{C} 2 \mathrm{C}$ are applicable in the case of e-Health in the following manner:

B2C- the capability of patients for online interaction with their healthcare systems.

B2B- improved possibilities for institution-to-institution transmissions of healthcare data for common benefit as evidence-based medicine.

C2C-new possibilities for communication and cooperation among similarly placed patients, especially with respect to self-management of chronic diseases.

The term "e" in e-Health does not stand for electronic alone, but many more e's. The author briefly described 10 e's related to e-Health: efficiency, enhancing quality, evidencebased, empowerment, encouragement, education, enabling, extending, ethics and equity [10].

\section{Contexts of e-Health Applications}

In an interview, Swedish e-Health strategist Henrik Ahlen expressed that currently, e-Health is used for meeting the challenges of growing healthcare needs from aging populations, increasing costs and staff shortages against increasing demands for faster and more personal access to healthcare (Widén \& Haseltine, 2015) [22]. Currently, most eHealth applications are used for fitness activities and self-care. This, experts say, is commendable but a far cry from where the world needs to be. To emphasize, e-Health is most beneficial in chronic patients. Available data shows that more than $75 \%$ of healthcare costs of countries are incurred by this group of patients. Therefore, cost reduction and efficiency and quality improvement will have a significant impact on national dimensions. Many e-Health initiatives have targeted this group patients already and e-Health is slowly finding its way into the way things are done.

\section{The Relevance OF E-HeAlth in SAUdi ARABia}

Saudi Arabia is a fast-developing nation. As the largest expporter of Crude Oil in the world and the centre of religious tourism globally, it wields quite a significant economic power among the comity of nations. When it comes to health, the nation is yet to fully come of age. Altuwaijri (2008) [6] noted that significant progress has been achieved in Saudi Arabia with respect to health care and some hospitals are now 
internationally recognised. However, its progress in e-Health had been very slow [10].

Almalki, FitzGerald \& Clark (2011) explain that e-Health and electronic information systems are already being implemented in hospitals and healthcare organisations like the King Faisal Specialist Hospital and Research Centre, the National Guard Health Affairs, Medical Services of the Armed Forces and the University Hospitals. Still e-Health systems are being implemented very slowly [5]. In $\mathrm{MOH}$ institutions, a number of information systems are operating in the regional directorates and the central hospitals. However, lack of coordination seriously affects the effectiveness of these systems.

From the previously discussed points, it would appear that some barrier exists for promoting e-Health in a big way. One possibility is that there is not enough knowledge about identifying system requirements and modelling of e-Health services to put into practice. This review tries to examine modelling and system requirements for e-Health services and how to adapt them to the Saudi conditions. Since e-Health is more relevant for care situations of chronic diseases, this review will make more references to discussions on e-Health models and requirements for chronic diseases care.

\section{MODELLING E-HEALTH SySTEM REQUIREMENTS}

Different authors have proposed many e-Health frameworks/systems. The components of these frameworks can be considered as the requirements of e-Health. Otherwise, the framework will not work. Some of the frameworks more relevant to Saudi Arabia are discussed below.

The chronic care model of Wagner (1998) given in Fig. 1 and the Innovative Care for Chronic Conditions Framework of WHO (WHO, 2002) given in Fig. 2 may be regarded as the most basic frameworks for e-Health systems dealing with chronic diseases. The WHO model is for universal applications, especially for developing countries, where healthcare is at a low level. The micro, meso and macro levels of the WHO model are the patients at the centre, the healthcare team and community partners, the broader healthcare organisation and community and the positive policy environment respectively. The self-management aspect comes under the micro level which is the patient.

The need to define the business goals of e-Health in a bid to identify system requirements of e-Health was emphasized by Alahmadi, Soh, and Ullah (2014). Most e-Health systems are successful in the pilot stage to the satisfaction of end users with regards to their initial objectives in terms of service quality. But very rarely do they proceed further to robust methods of daily practice. The excessive focus on technology in these models is not helpful in developing the kind of understanding needed between stakeholders and developers for success to be achieved. This had been the cause for failure of many e-Health projects. Besides system requirements and value propositions, what is required or expected to provide the particular service needs to be clearly stated. Different stakeholders may require varying e-Health goals and values as needs and objectives differ. This complicates the goal-setting task. Therefore, it becomes increasingly necessary to identify and reconcile all the system and stakeholder requirements in the very beginning. This is where requirement engineering comes to play. The term "requirements engineering" is the software engineering aspect that has to do with the processes of identifying, documenting, recording and maintaining the system through the identification of stakeholders and their needs, which can, then, be analysed, communicated and implemented subsequently. The three main categories of requirements engineering are (i) real-world goals (ii) functions, and (iii) constraints. These axes determine the development of the system based on the description of "what" and "why". There are four steps in system requirements engineering. These are (i) requirements elicitation (ii) requirements negotiation (iii) requirements specification, and (iv) requirements validation. The author provides a methodology to understand the e-Health business environment and come up with system requirements based on organisational goals. Without a clear understanding of the environment, it may be difficult (if not impossible) to efficiently arrive at business goals. It is these goals that will then aid the identification of e-Health system requirements. There are two main phases to this methodology, as illustrated in Fig. 3.

Phase 1 of this framework deals with the well-known and accepted business/IT alignment approach. There are three levels in this phase;

Level 1- e-Health organisation. This makes up the decision level for any e-Health business environment. It describes the details of the aims and objectives of the organisation, the available resources to achieve those objectives and the executives.

Level 2- e-Health business strategy. Once you have identified the objectives and resources, this level goes on to provide a definite, practical and responsive strategy and plan for the e-Health business. This must be encompassing though, and should involve major stakeholders and sectors. The health goals and targets, vision and evaluation, and general objectives of the e-Health strategy are clearly defined here.

Level 3- e-Health business infrastructure. This is where operations take place. It forms the foundations for services and the exchange of information beyond geographical and sectoral boundaries for the e-Health programme. Various dimensions of operations include the physical infrastructure, core services, processes and activities. While Phase 2 dealth with the eHealth business goals modelling methods as they concern eHealth system requirements, this phase elicits the e-Health system requirements using the business goals identified in Phase 1.

The author used a routine patient visit to a healthcare unit in Australia as a case study to illustrate the model of the eHealth system environment using business process modelling symbols. Registration, doctor allocation, consultation, examination as well as discharge are the four processes and activities within the e-Health model. From this model, the method of eliciting system requirements as given in Fig. 4.

In another related paper, the same authors (Alahmadi, Soh, \& Ullah, 2014) proposed a model of automated e-Health 
system for a patient visit to the hospital in Australia. To elicit the requirements for such system, first tiers of the system were used [1]. Tier 1 consists of e-Health environment in which goals, subgoals and activities exist. In Tier 2, automated eHealth system requirements are derived. These are diagrammatically explained by the authors as given in Fig. 5. Clearly, it is more convenient to elicit system requirements as an end-product of activities in Tier 2.

To address privacy issues, an accountable e-Health system $(\mathrm{AeH})$ was designed by Gajanayake, Iannella, Lane, and Sahama (2012) in Australia. The model is reproduced in Fig 6. In this model, there are three types of users: a central health authority, patients, and healthcare providers (HCP). The accessibility for each user is determined by the need and justification separately. The governing body of HER determines the levels of its employees who can access the patient records in a role-based manner. The patients may access his/her data or nominate anyone else [12]. These two access policies are combined by a pre-determined protocol between the patient and the HCP. The patient can question the HCP if any misuse occurs. The authors presented the same system as a use case diagram.

A business continuity model for e-Health systems was proposed by Rejeb, Bastide, Lamine, Marmier, and Pingaud (2012) so that the system remains operative even in unexpected conditions. A protected backup system is used for this purpose [17].

A quality of experience (QoE)/quality of service (QoE) model was proposed by Rojas-Mendizabal, Serrano-Santoyo, Conte-Galvan, and Gomez-Gonzalez (2013) based on an eHealth ecosystem consisting of human, technological and economic contexts. The QoE/QoS model is reproduced in Fig. 7.

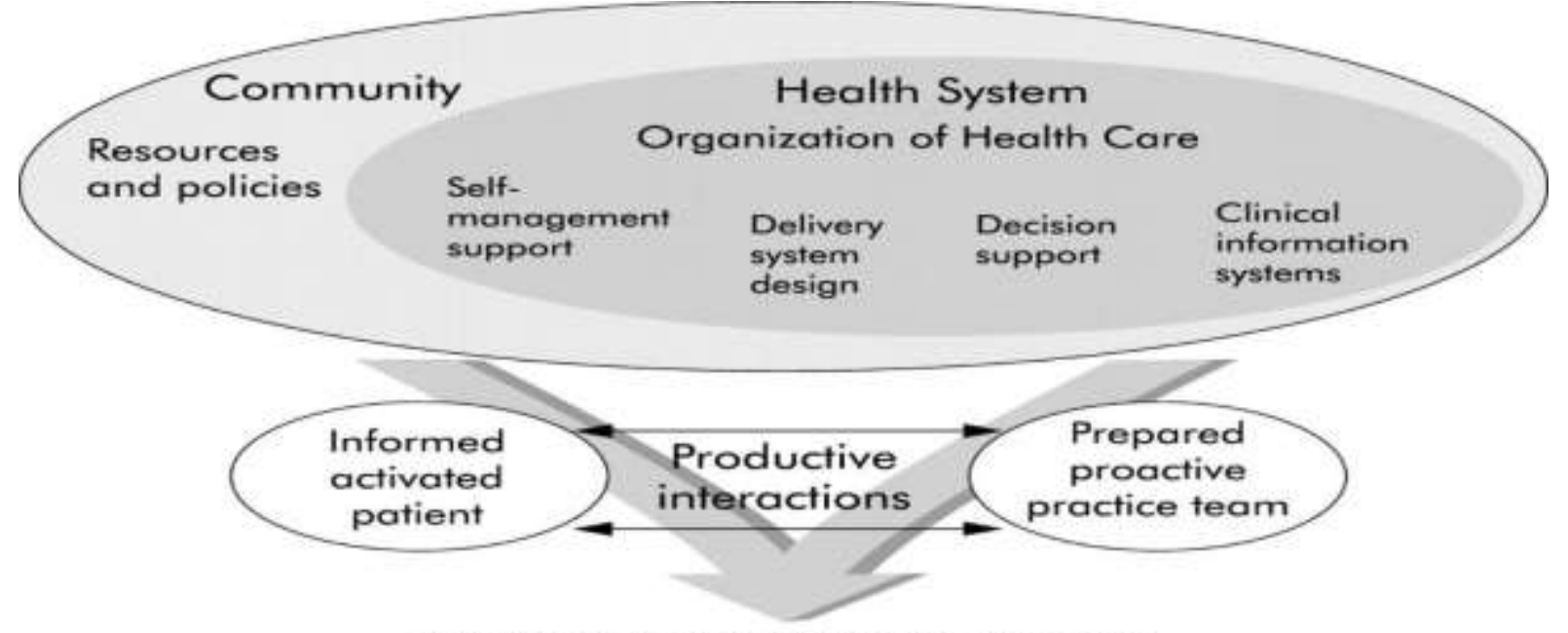

\section{Functional and clinical outcomes}

Fig. 1. The Chronic Care Model of Wagner (1998).

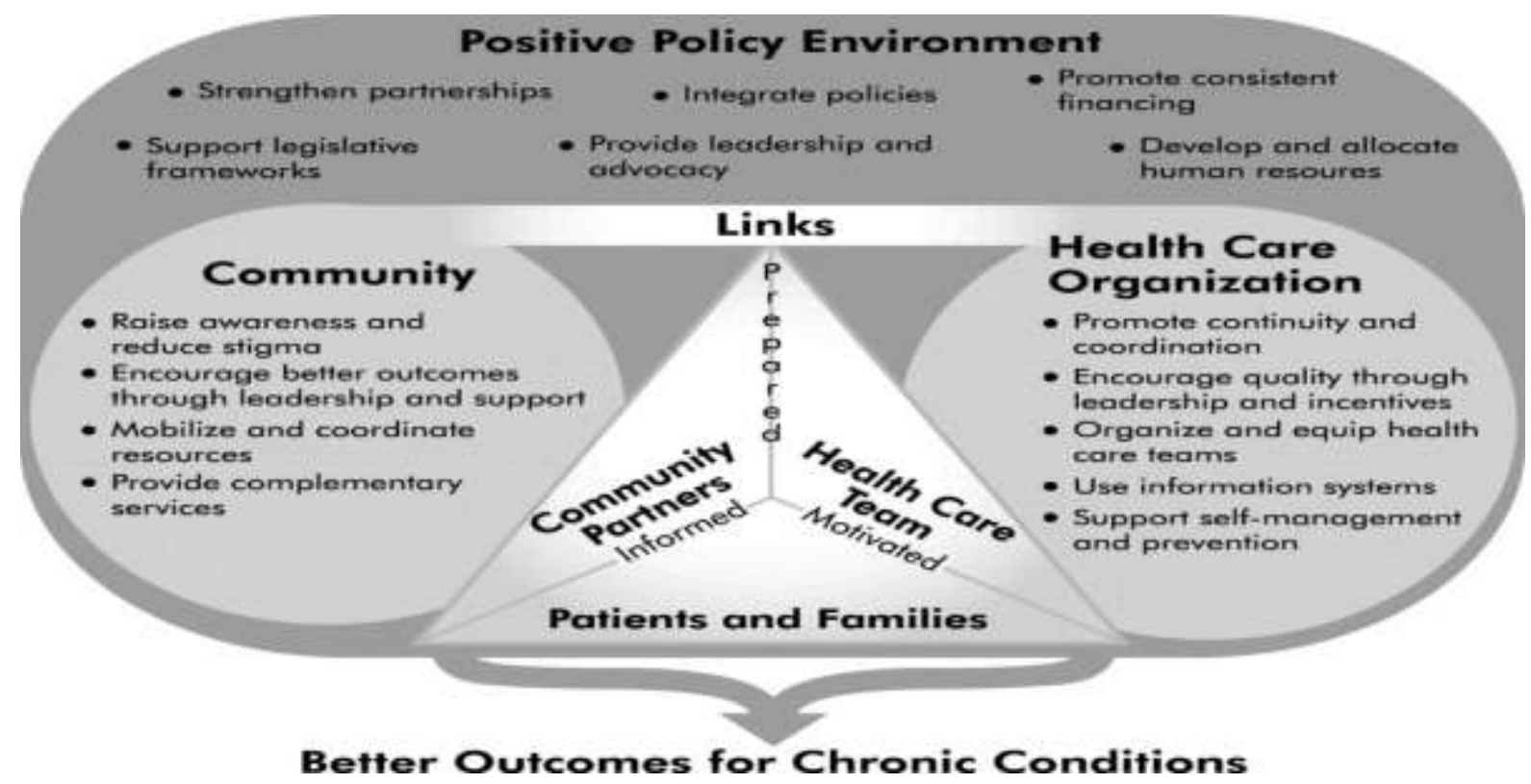

Fig. 2. Innovative Care for Chronic Conditions Framework (WHO, 2002) [21]. 


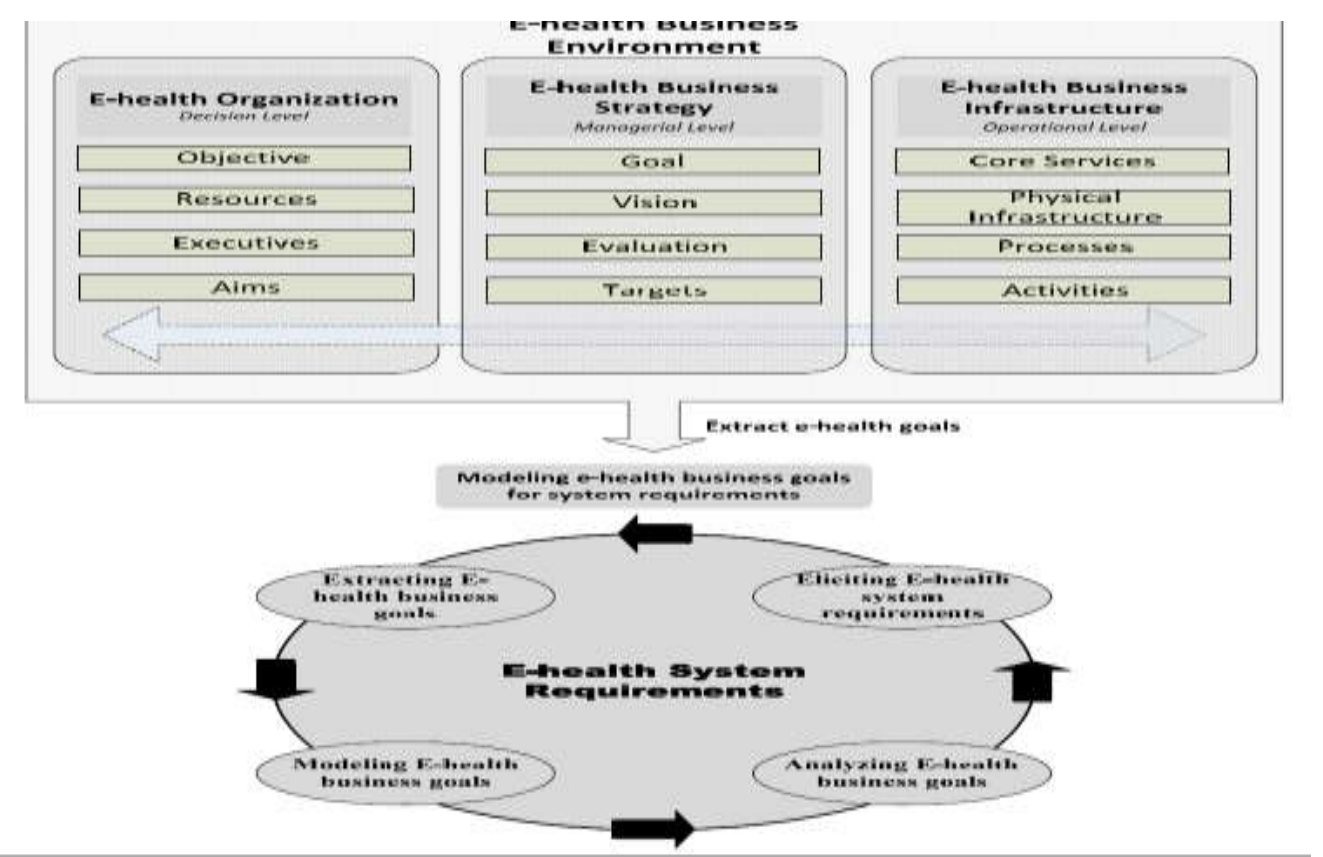

Fig. 3. Methodology of Deriving System Requirements from Business Goals in an e-Health Environment (Alahmadi, Soh, \& Ullah, 2014) [2].

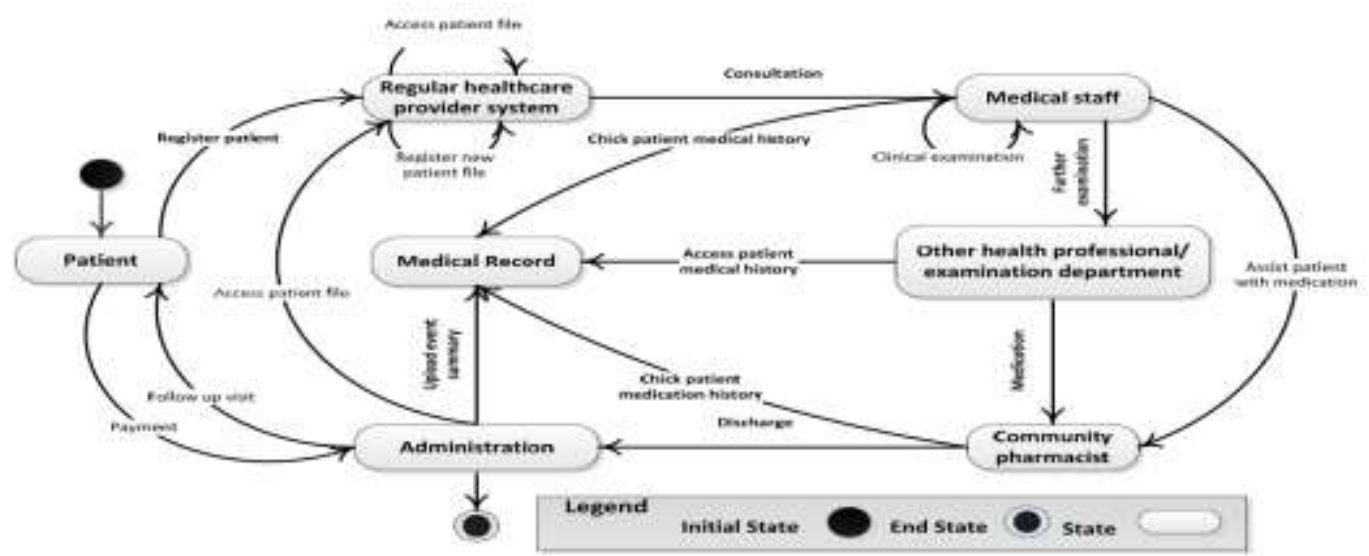

Fig. 4. Method of Extracting e-Health System Requirements based on e-Health Environment Modelling (Alahmadi, Soh, \& Ullah, 2014).

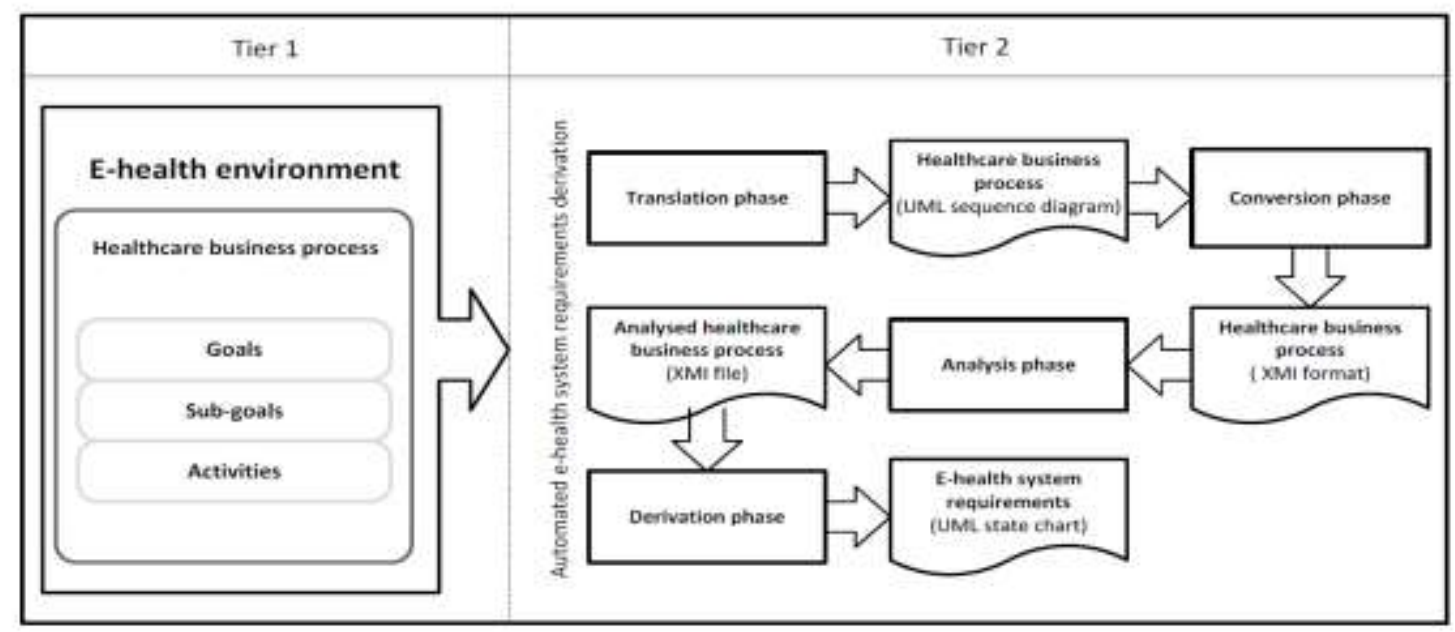

Fig. 5. Automatic e-Health Modelling Approaches (Alahmadi, Soh, \& Ullah, 2014). 


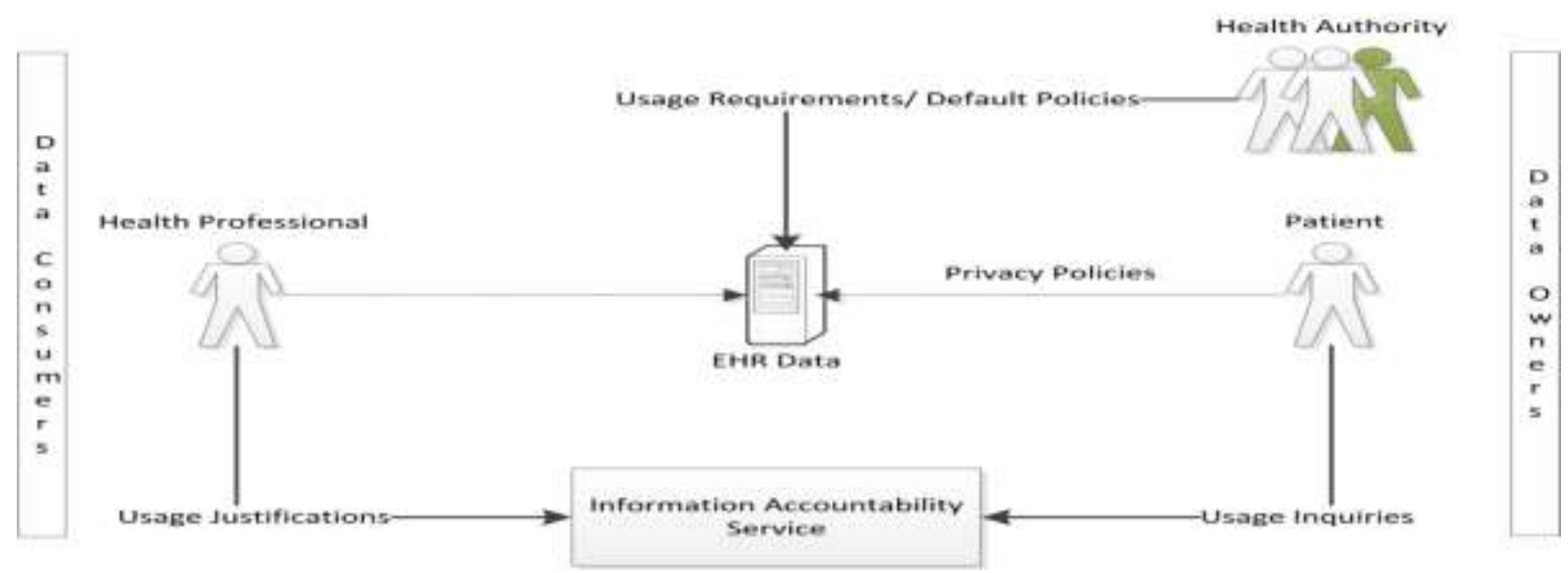

Fig. 6. An Accountable e-Health Model (Gajanayake, Iannella, Lane, \& Sahama, 2012).

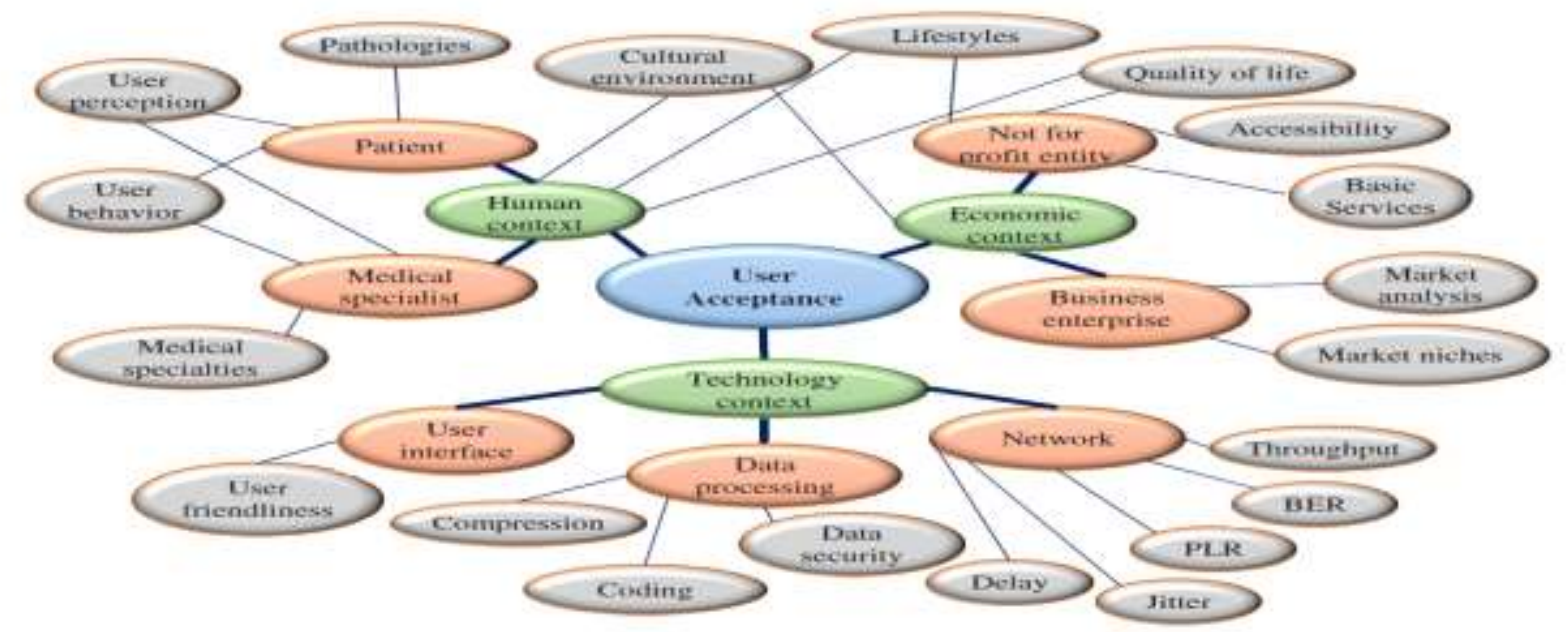

Fig. 7. A QoE/QoS e-Health Model Proposed by (Rojas-Mendizabal, Serrano-Santoyo, Conte-Galvan, \& Gomez-Gonzalez, 2013) [19].

A behaviour intention technology (BIT) model for eHealth and mobile e-Health (m-health) applications was proposed and applied to a fitness case by Mohr, Schueller, Montague, Burns, and Rashidi (2014) [16]. The basic model is reproduced in Fig. 8. BIT models use a variety of technologies such as sensors, mobile phones and the web to support users to change behaviours and cognitions of physical health, mental health and wellness problems and interventions to reduce them. The basic requirement is effecting a behavioural change to promote a change from current state of health to a better state of health for the user. Preparation of workflows of such models will be useful to determine when the intervention should be applied. Predetermined or adaptive technological systems can be used depending upon the context.

A service-oriented boundariless e-Health architecture was proposed and applied to an application, CardioNet by Mircea, et al. (2010). It is a patient-centric model, in which, all hardware, software and medical activities are services and patients are customers. This is a truly business model as well. Remote interactions are possible between patients, doctors, providers, labs and authorities. The possible range of interactions is depicted in Fig. 9. Data exchange between the interacting elements occurs through XML format. How it is implemented in the CardioNet is shown in Fig. 10 reproduced from the work. In CardioNet, boundaryless information flow occurs at the levels of patient, provider, operational centres, home care units and data storage devices.

An RFID based rural e-Health service model was proposed by Chia, Zalzala, Zalzala, and Karim (2013). The hierarchical structure constitutes rural inhabitants (patients), community health workers and the central healthcare facility. The goal of such an e-healthcare system is to facilitate easy and reliable identification of each patient, maintain more precise medical records, provide better healthcare and improve the quality of life in communities that are remote from a central medical facility. It also reduces the pressure on the central healthcare facility [9]. The patient base of the central facility is broadened towards remote localities. The value chain stakeholders are the patients, other inhabitants of the rural area, physicians, administrators of the central facility and providers of equipment, e-Health recorder and communication facilities. The actual rural e-Health system is reproduced in Fig. 11. 


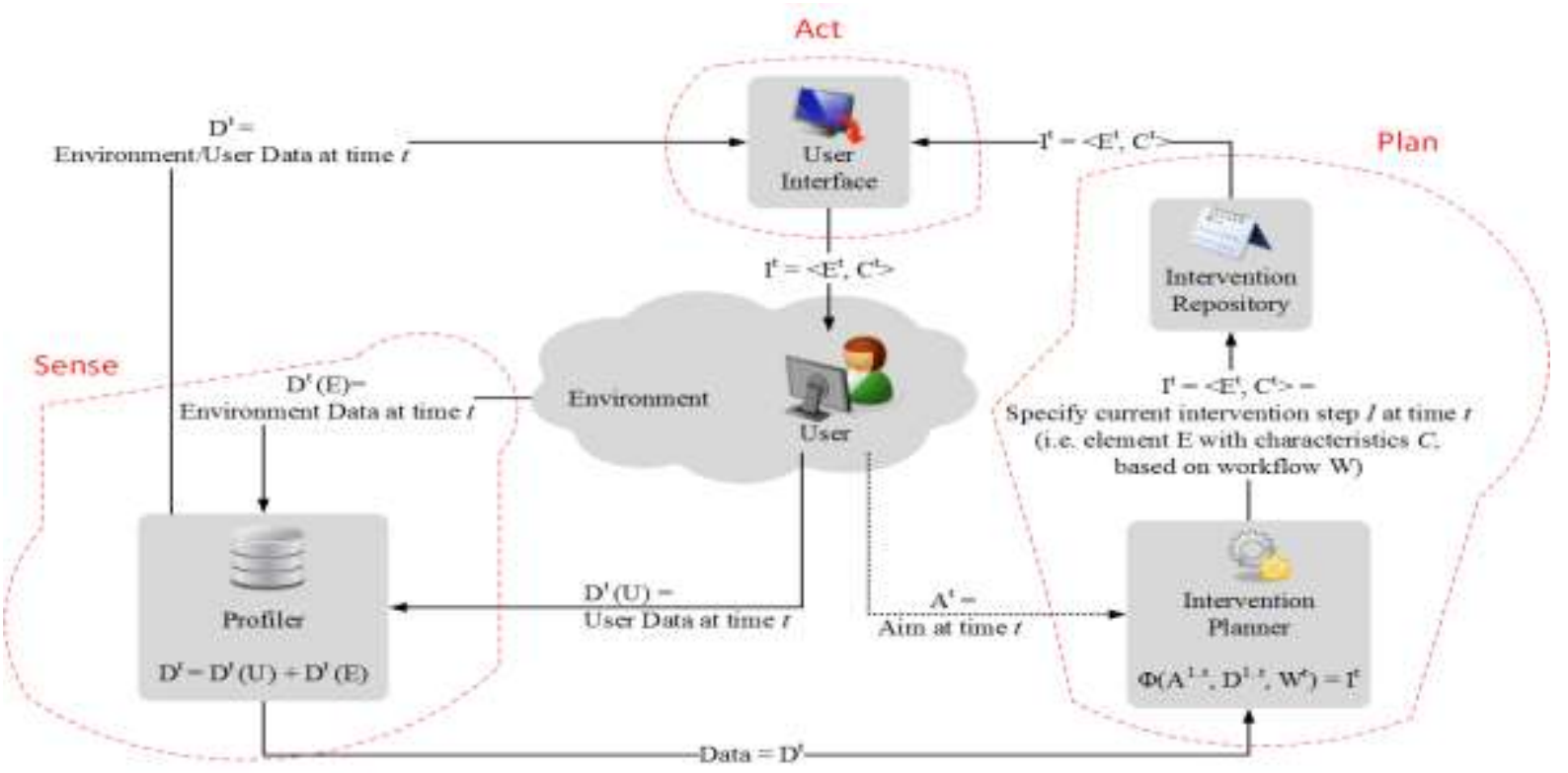

Fig. 8. A basic BIT e-Health Model (Mohr, Schueller, Montague, Burns, \& Rashidi, 2014).

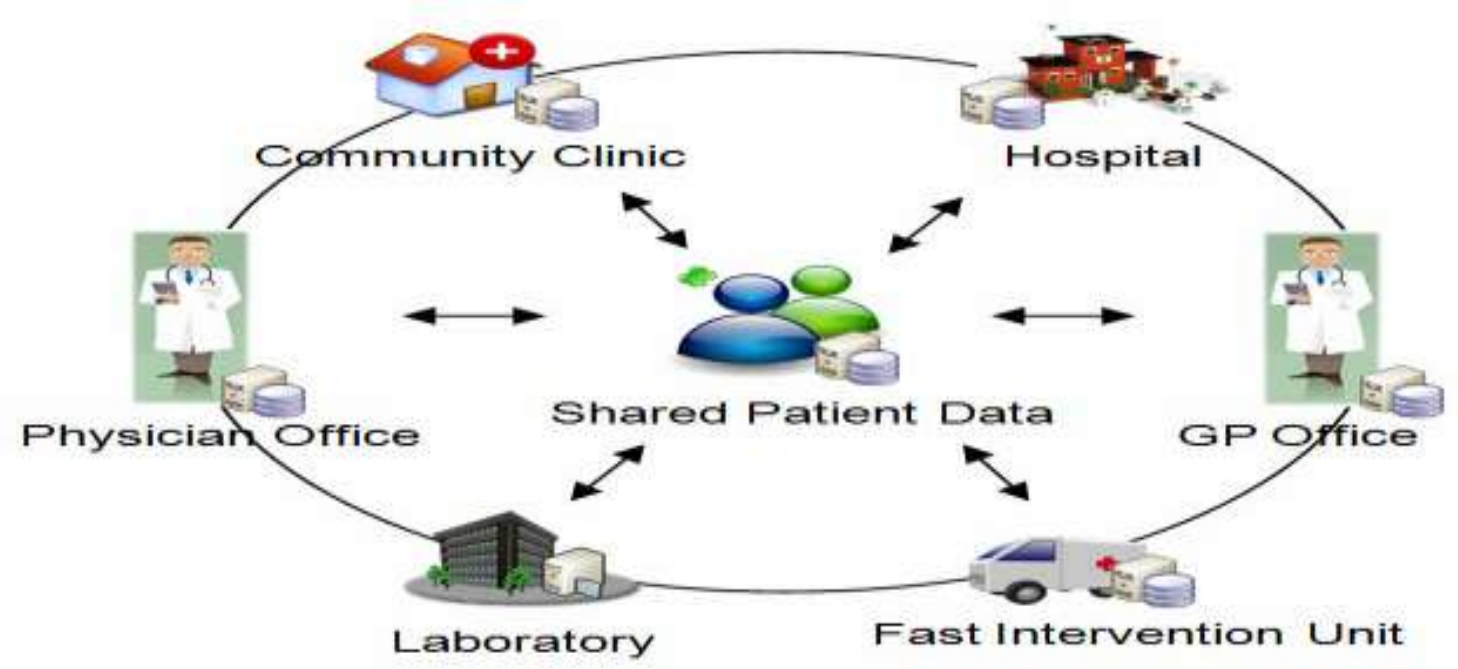

Fig. 9. Boundaryless Information Flow (Mircea, et al., 2010) [15].

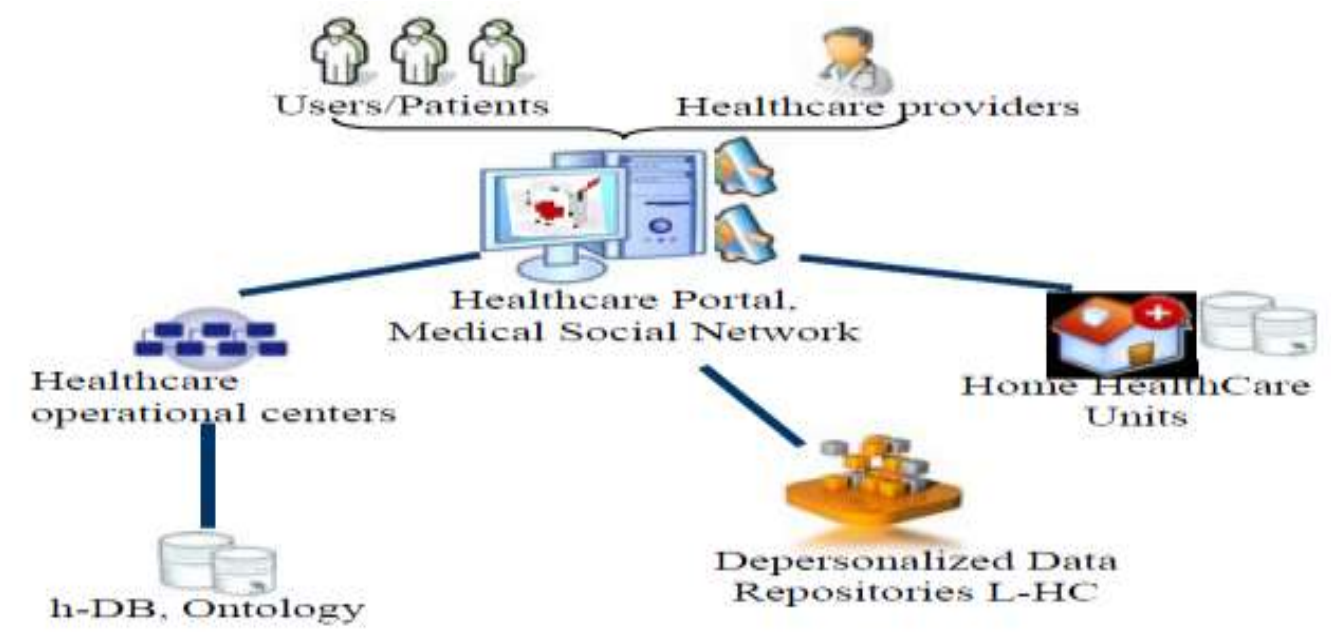

Fig. 10. Application of Boundaryless Information Flow in CardioNet (Mircea, et al., 2010). 


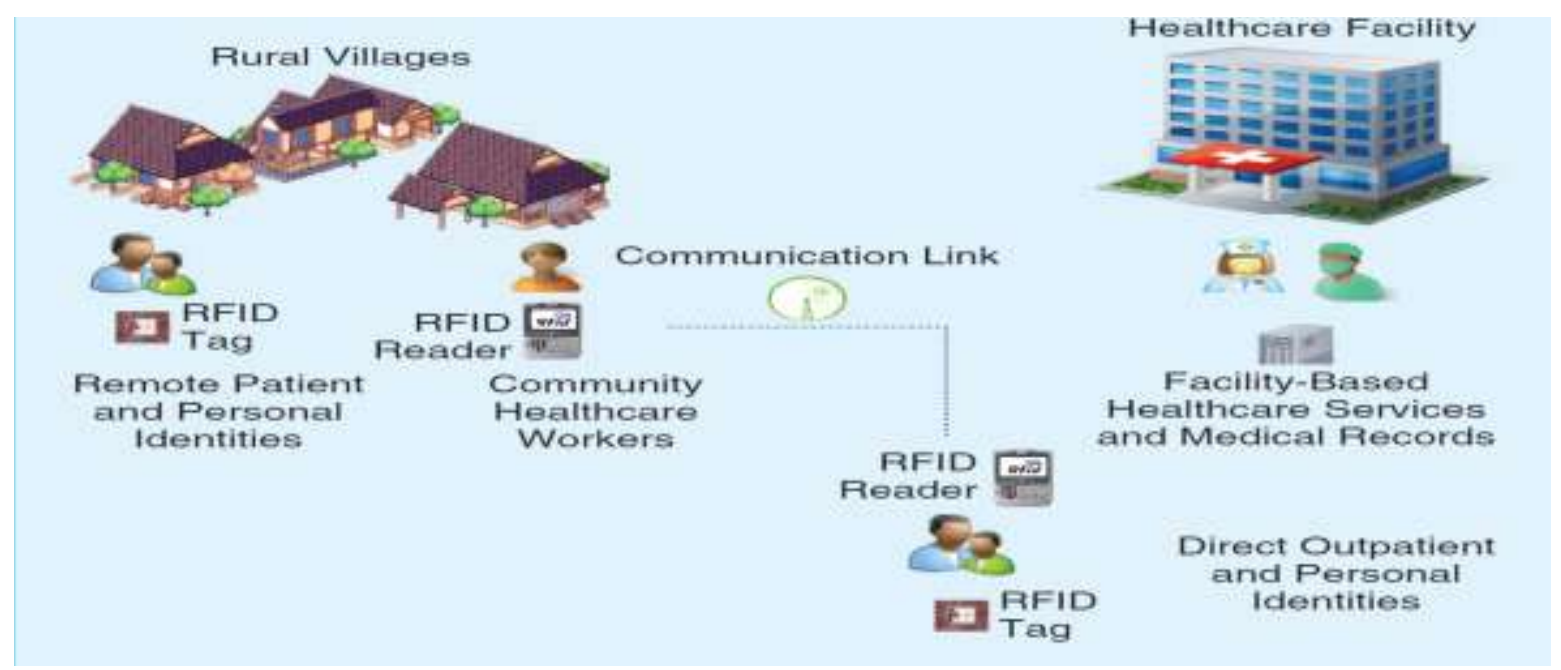

Fig. 11. RFID-based Rural e-Health System (Chia, Zalzala, Zalzala, \& Karim, 2013).

The system model and security requirements of a secure patient-centric e-Health model was defined by Barua, Liang, $\mathrm{Lu}$, and Shen (2011). The proposed model has the e-Health care service provider working as a trusted party where a patient is registered. The encrypted data is stored in centralised storage, a sort of health-cloud from where future access is possible. The proposed scheme has four major steps: (i) the patient health information (PHI) is collected (ii) the secure encrypted data is transmitted from PHI to e-healthcare provider (iii) at the e-Health provider, the PHI data are processed. Then it classifies the PHI data based on the patientpreferred attributes. Access to the data is based on different privacy levels of requesters like general users, doctors etc. by assigning a different set of attributes to the different levels, and (iv) the processed PHI is transferred to the cloud for future use [7]. The security requirements were defined based on the goals: patient-centric access control, integrity of the message using authenticated sources and non-repudiation, prevention of attacks by cyphertext only methods, provision of patient safety, resistance to collusion attack and resistance to DoS attacks. For each of the security goals, requirements and technologies were developed and integrated into the ehealthcare system.

In his work on implementation of a cloud-based e-Health services in an Indian state, (Joshi, 2016) diagrammatically presented the following requirements-

1) Software as a service (SaaS) to citizens through government portals, mobile phones, computers, self/start architecture and m-health applications. These are public services.

2) Public/private services of Platform as a Service (PaaS) for database, reporting, multitenancy and programming languages. These provide PaaS for SaaS development.

3) Infrastructure as a Service (IaaS) for local units statewide and servers for database, web and applications are provided with the virtual environment for users to operate. SWAN can be used for connecting various health departments [14].
This list can be used as the basic system requirements of eHealth projects in general. The authors also suggested some challenges and solutions.

\section{A. Discussion}

The different e-Health models and requirements discussed above focus on salient issues that often come up when the subject of e-Health is brought up. Take Wagner's Chronic Care model for instance, it is a framework for chronic diseases that focuses on the patient, healthcare team and community partners in developing countries where access to quality healthcare is still a major challenge. Focusing on micro, meso and macro approaches, the primary aim of this model is to ensure that healthcare delivered electronically is supported by a wide coordinated approach cutting across levels of government. The Innovative Care for Chronic Conditions Framework introduced by the WHO in 2002 follows virtually the same approach.

To address privacy issues which are likely to come up when dealing with medical records, an Accountable e-Health System was proposed. As clearly explained above, this addresses the issues of who has access to what; should a patient's medical records be left out in the open? How do you determine who can see them? What about protecting them from just anybody? In many countries, including Saudi Arabia, there is still the long-drawn debate about how PatientDoctor confidentiality will be maintained in an e-Health system. This model answers that question with a proposition that involves determining access based on need and justification. It is also a flexible system that allows a patient to nominate anyone that can access his health information. For health providers, employees are categorized according to levels and access are granted to only those who truly "need" such access.

Chia et al (2013) proposed a Radio Frequency Identification (RFID) rural e-Health model that seeks to have a common database of residents in an area based on their health status. This is quite a model because of its focus on rural health. Rural settlers across the world have been at the wrong side of access to healthcare for decades. These 
residents often encounter barriers that prevent them from getting the healthcare they need (Rural Health Information Hub, 2020). To change this, there is need to provide access to appropriate and necessary healthcare services for rural residents. With this proposed model, this goal can be achieved. This model fails, however, to consider availability of RFID systems in rural communities and whether residents have the tools and knowledge required for such a model to work.

The other models highlighted in this study may focus on several other specifics, but the bottom line is the search for model requirements for e-Health application across the world.

\section{LIMITATIONS}

As a contribution to the existing body of research that seeks an efficient solution to implementing e-Health in societies, this study, and specifically, to identify an e-Health system modelling requirement that suits the Saudi Arabia health system, this study relies heavily on previous works of literature on the subject. However, in the area of a truly specific model for Saudi Arabia, this is a virgin attempt. Not many scholars (if any at all) have attempted a study of eHealth modelling requirements in a bid to find one that suits the Saudi Arabian experience. Furthermore, much of the literature and technologies studied are not peculiar to Saudi Arabia as not much has been done in that regard. The author only sought to examine how, learning from the experiences and models proposed for other climes, a model and its requirements can be developed for the Saudi Arabian nation, which is what this study attempts to do.

\section{WHAT ARE THE MODELLING SySTEM REQUIREMENTS FOR E-HEALTH IN SAUDI ARABIA?}

The above review shows the possibility of multiple contexts and models of e-Health using technologies like cloud, RFID and mobile phones. Evidently, these varied contexts, models and technologies have different system requirements. Also, chronic health conditions seem to be the best contexts for e-Health application according to some of the proponents.

In the case of Saudi Arabia, diabetes and obesity are the most serious chronic health conditions even within the younger demographics due to lifestyle habits of food and nutrition as well as physical activity. In Saudi Arabia, as per the International Diabetes Federation, out of the population of 20.77 million, 3.852 million adults $(18.5 \%)$ had diabetes in 2017 (IDF, 2019) [13]. News (2019) cited Colliers Report of $17.9 \%$ diabetes and another $35.4 \%$ obese adults in Saudi Arabia. As the prevalence rapidly increases through an increase in the incidence rates among children and adolescents, especially Type 1 diabetes (Robert, Al-Dawish, Mujammami, \& Al Dawish, 2018) [18], proper data collection systems in regular frequencies need to be put in place.

The high prevalence rate of diabetes in Saudi Arabia shows that management of diabetic patients and control of the disease is a serious problem. The average annual cost of diabetes treatment without complications is $\$ 2600$ for each Type 1 Diabetes (T1D) patient and about $\$ 2000$ per Type 2 Diabetes (T2D) patient, according to an estimate given by the
Saudi Ministry of Health. The country cost burden for diabetes treatment may reach $\$ 6.5$ billion by 2020 . Presenting these statistics, Alanzi (2018) noted that policy deficits and scarce research on e-Health systems for diabetes have resulted in its poor implementation at hospital level. The e-Health initiatives of the Saudi Ministry of Health were limited to creating silos of health records and some service facilitations [3]. There had been only some very recent works on this issue in Saudi Arabia like those of Aldahmash, Ahmed, Qadri, Thapa, and AlMuammar (2019) and Belcher, Vess, and Johnson (2019) [4] [8].

It is often better to resort to self-management of these problems after initial stages of medical consultations. This will help to reduce the pressure on care hospitals in terms of resources and human power. In self-management, it is possible to obtain the help of community health workers, paramedical professionals and diabetes educators to help from time to time to ensure that the correct procedures are followed. The system does not preclude consultation with specialists if things go out of hand. However, this cannot be effective without a proper eHealth system in place that provides the information resources and monitoring mechanisms needed for self-management.

As many of the reviewed works above show, goal setting is the first step to model the system, based on which the requirements can be derived. In determining the modelling system requirement for patient self-management of diabetes, these steps will be discussed below.

\section{A. Goal setting}

1) Self-management goals-

a) Patient health records before self-management start.

b) The doctor gives the advice for self-management of blood glucose, medication, diet control, obesity (BMI), exercise and any other activity.

\section{2) Self-management process-}

a) Self-monitoring of blood glucose, diet and physical activity as per the schedule and methods prescribed by the doctor. Assistance of local community health worker can be sought.

b) The observed values are entered in the patient's page of the e-Health website for further processing, cloud storage and access as per requirement.

c) The website sends alert signals to the doctor for advice to the patient if any entered value is out of control.

d) Whether the patient complies with the advice can be known from the subsequent monitoring values.

3) National goals-

a) Nationwide screening of population for diabetes and obesity based on WHO criteria.

b) Maximum coverage of diabetic and obese people across the country for e-Health applications.

c) Creation of electronic health records of all affected persons, whose records are not already available.

d) Processing of the electronic data and storing in a PHI.

e) Data encrypted and stored in cloud. 
f) Access to data to stakeholders as per their privacy levels, determined based on role and necessity.

g) Adequate security of the data.

h) System requirements

4) Based on the above goals, the system requirements are as follows-

a) Infrastructure- Website, hardware, software, servers, cloud systems, networking of hospitals and communities, remote access, as well as fast and efficient internet service throughout the country.

b) Resources- Finance, buildings and related equipment. Supplies of medication including insulin injection, monitoring devices, exercise outfits, and other needed materials.

c) Human power- Healthcare professionals in hospitals and community health workers, diabetes educators at community levels also required.

d) Technology- Hardware and software, servers, Internet, cloud, PaaS, SaaS, IaaS, cybersecurity and other required technology.

e) Administration and management- Coordination by a dedicated team of $\mathrm{MOH}$ management.

f) Regular monitoring and evaluation of the e-Health system for effectiveness by the management and coordination team.

\section{Conclusions}

An examination of some selected literature revealed the essential steps to be followed for identification of modelling system requirements for e-Health systems. Based on these steps, a preliminary attempt has been made for identification modelling system requirements for e-Health application for the serious chronic problem of diabetes (and obesity) selfmanagement by patients in Saudi Arabia. These modelling system requirements were arrived at based on identified goals for managing diabetes and obesity in Saudi Arabia. The model is only a skeletal approach and an attempt based on available information. It will require subsequent studies for refinement and testing before large scale implementation can be considered and carried out.

\section{REFERENCES}

[1] H. Alahmadi, B. Soh, and A. Ullah, (2014). "Automated health business process modelling and analysis for e-health system requirements elicitation". Pacific Asia Conference on Information Systems (PACIS) 2014 Proceedings (p. 13 pp). AIS Electronic Library (AISeL). Retrieved October 30, 2019, from https://pdfs.semanticscholar.org/1e3d/d7fc30aec 3c3fcac6e41ece710413fc291bf.pdf.

[2] A. H. Alahmadi, B. Soh, and A. Ullah, (2014). Improving e-Health Services and System Requirements by Modelling the Health Environment. Journal of Software (JSW), 9(5), 1189-1201. doi:10.4304/jsw.9.5.1189-1201.

[3] T. Alanzi, (2018). mHealth for diabetes self-management in the Kingdom of Saudi Arabia: barriers and solutions. Journal of $\begin{array}{lll}\text { Multidisciplinary } \quad \text { Healthcare, } & 11, & 535-546 .\end{array}$ doi:10.2147/JMDH.S174198.

[4] A. M. Aldahmash, Z. Ahmed, F. R. Qadri, S. Thapa and A. M. AlMuammar, (2019). Implementing a connected health intervention for remote patient monitoring in Saudi Arabia and Pakistan: explaining 'the what'and 'the how'. Globalisation and health, 15(1), 20. doi:10.1186/s12992-019-0462-1.
[5] M. Almalki, G. FitzGerald and M. Clark, (2011). Health care system in Saudi Arabia: an overview. Eastern Mediterranean Health Journal, 17(10), 784-793. Retrieved October 29, 2019, from https://apps.who.int/iris/bitstream/handle/10665/118216/17_10_2011_07 84_0793.pdf.

[6] M. M. Altuwaijri, (2008). Electronic-health in Saudi Arabia. Just around the corner? Saudi medical journal, 29(2), 171-178. Retrieved October 29, 2019, from https://europepmc.org/abstract/med/18246222

[7] M. Barua, X. Liang, R. Lu and X. Shen (2011). Peace: An efficient and secure patient-centric access control scheme for ehealth care system. IEEE Conference on Computer Communications Workshops (INFOCOM WKSHPS), 10-15 April 2011, Shanghai, China (pp. 970975). IEEE. doi:10.1109/INFCOMW.2011.5928953.

[8] T. Belcher, J. Vess and E. Johnson (2019). Using patient portal messaging to improve glycemic control in adult patients with diabetes in Saudi Arabia. Online Journal of Nursing Informatics (OJNI), 23(1). Retrieved June 21, 2019, from https://www.himss.org/library/usingpatient-portal-messaging-improve-glycemic-control-adult-patientsdiabetes-saudi-arabia.

[9] S. Chia, Zalzala, L. Zalzala and A. Karim, (2013). Intelligent technologies for self-sustaining, RFID-based, rural e-health systems. IEEE Technology and Society Magazine, 32(1), 36-43. Retrieved October 30, 2019, from http://lifesciences.embs.org/wpcontent/uploads/sites/53/2013/05/06479445.pdf.

[10] G. Eysenbach, (2001). What is e-health? Journal of medical Internet research, 3(2), e20. doi:10.2196/jmir.3.2.e20

[11] F. Alanezi, Factors affecting the adoption of e-health system in the Kingdom of Saudi Arabia, International Health, , ihaa091, https://doi.org/10.1093/inthealth/ihaa091.

[12] R. Gajanayake, T. Alannella, B. Lane and T. Sahama, (2012). Accountable-ehealth systems: The next step forward for privacy. Proceedings of the 1st Australian eHealth Informatics and Security Conference, held on the 3rd-5th December, 2012 at Novotel Langley Hotel, Perth, Western Australi (pp. 22-28). Edith Cowan University. doi:10.4225/75/5796fa8940a98.

[13] IDF. (2019). Middle East and North Africa. Retrieved June 20, 2019, from International Diabetes Federation: https://www.idf.org/ournetwork/regions-members/middle-east-and-north-africa/members/46saudi-arabia.html.

[14] M. Joshi, (2016). Proposed cloud based Framework for Implementing EHealth Services in Uttarakhand. Proceedings of International Conference on Advanced Computing (ICAC-2016) (pp. 367-371). College of Computing Sciences and Information Technology (CCSIT), Teerthanker Mahaveer University, Moradabad. Retrieved October 30, 2019, from $\mathrm{http} / / / \mathrm{tmu}$.ac.in/college-of-computing-sciences-and-it/wpcontent/uploads/sites/17/2016/10/ICAC-1604227.pdf.

[15] R. U. Mircea, G. Saplacan, G. Sebestyen, N. Todor, L. Krucz and C. Lelutiu, (2010). eHealth: towards a healthcare service-oriented boundary-less infrastructure. Applied Medical Informatics, 27(3), 1-14. Retrieved October 30, 2019, from https://ami.info.umfcluj.ro/index. $\mathrm{php} / \mathrm{AMI} /$ article/view/28.

[16] D. C. Mohr, S. M. Schueller, E. Montague, M. N. Burns and P. Rashidi, (2014). The behavioral intervention technology model: an integrated conceptual and technological framework for eHealth and mHealth interventions. Journal of medical Internet research, 16(6), e146. doi:10.2196/jmir.3077.

[17] O. Rejeb, R. Bastide, E. Lamine, F. Marmier and H. Pingaud, (2012). A model driven engineering approach for business continuity management in e-Health systems. 6th IEEE International Conference on Digital Ecosystems and Technologies (DEST), 18-20 June 2012, Campione d'Italia, Italy (pp. 1-7). IEEE. doi:10.1109/DEST.2012.6227931.

[18] A. Robert, A. Al-Dawish, M. Mujammami and M. A. Al Dawish, (2018). Type 1 diabetes mellitus in Saudi Arabia: a soaring epidemic. International journal of pediatrics, ID 9408370, 9 pages. doi:10.1155/2018/9408370.

[19] V. A. Rojas-Mendizabal, A. Serrano-Santoyo, R. Conte-Galvan and A. Gomez-Gonzalez, (2013). Toward a Model for Quality of Experience and Quality of Service in e-health Ecosystems. Procedia Technology, 9, 968-974. doi:10.1016/j.protcy.2013.12.108. 
[20] Salah, Al-Sharhan, Omran, Esraa and K. Lari, (2018). An integrated holistic model for an eHealth system: A national implementation approach and a new cloud-based security model. International Journal of Information Management, 47 (pp.121-130). Retrieved January 9, 2021 from https://www.sciencedirect.com/science/article/abs/pii/S02684012 17305273.
[21] WHO. (2019). eHealth at WHO. Retrieved June 20, 2019, from WHO: https://www.who.int/ehealth/en/.

[22] S. Widén and W. A. Haseltine, (2015). eHealth Technologies and the Future of Healthcare: Interview with Henrik Ahlen. Access Health International, . Retrieved June 20, 2019, from https://accessh.org/wpcontent/uploads/2015/12/Henrik-Ahlen1.pdf.

APPENDIX

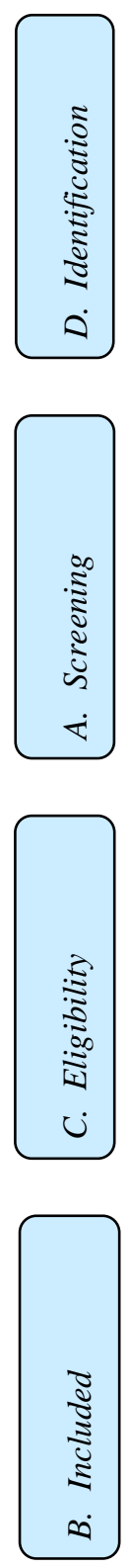

Records identified through database searching $(\mathrm{n}=74)$
Additional records identified through other

$$
\text { sources }
$$$$
(\mathrm{n}=0)
$$

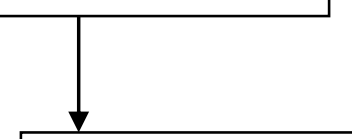

Records excluded

$$
(n=6)
$$

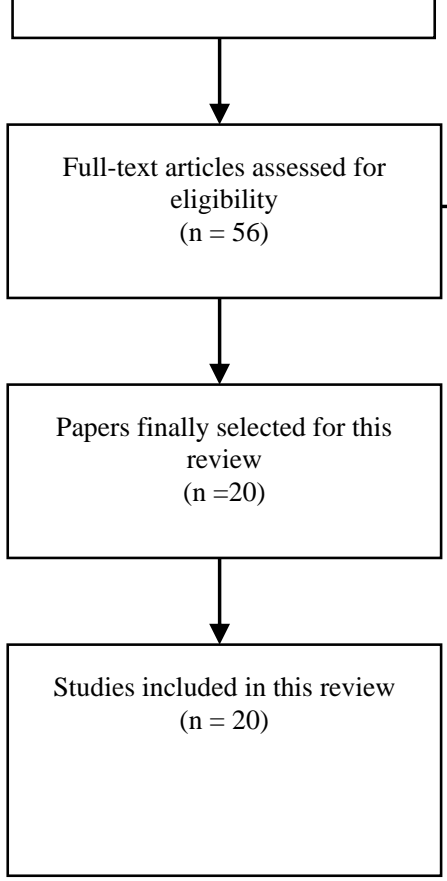

Full-text articles excluded, with

$$
\text { reasons }
$$

PRISMA 2009 Flow Diagram 